\title{
ON THE CONCEPT OF OPTIMALITY INTERVAL
}

\author{
LLUÍS BIBILONI, PELEGRÍ VIADER, and JAUME PARADÍS
}

Received 18 January 2001 and in revised form 15 June 2001

\begin{abstract}
The approximants to regular continued fractions constitute best approximations to the numbers they converge to in two ways known as the first and the second kind. This property of continued fractions provides a solution to Gosper's problem of the batting average: if the batting average of a baseball player is 0.334 , what is the minimum number of times he has been at bat? In this paper, we tackle somehow the inverse question: given a rational number $P / Q$, what is the set of all numbers for which $P / Q$ is a best approximation of one or the other kind? We prove that in both cases these optimality sets are intervals and we give a precise description of their endpoints.
\end{abstract}

2000 Mathematics Subject Classification: 11J70.

1. Introduction. The connection between continued fractions and best approximations is well known. It seems that Huygens (1629-1695) was the first to realize that the approximants of a continued fraction show the property that can be described as "best approximation" to a number, see [1, page 86]. The following two definitions of best approximation are from Khintchine's continuous fractions [4, pages 27-35].

DEFINITION 1.1 (Khintchine [4]). (a) A rational fraction $P / Q$ is a best approximation of the first kind to the real number $\omega$, if any other rational fraction having the same or smaller denominator differs from this number more than $P / Q$. In symbols,

$$
\left|\omega-\frac{p}{q}\right|>\left|\omega-\frac{P}{Q}\right|
$$

whenever $p / q \neq P / Q, 0 \leq q \leq Q$.

(b) A rational fraction $P / Q$ is a best approximation of the second kind to the real number $\omega$, if from $p / q \neq P / Q, 0 \leq q \leq Q$ there follows

$$
|q \omega-p|>|Q \omega-P| \text {. }
$$

A best approximation of the second kind is also known as an optimal approximation.

REMARK 1.2. Best approximations of the second kind sometimes behave in a rather nonintuitive way. For instance, it may happen that $p / q<P / Q<\omega$ while $p / q$ approximates $\omega$ better than $P / Q$.

The motivation for this paper comes from an interesting problem by Bill Gosper cited in [5, Example 39, page 363].

If a baseball player's batting average is 0.334 , what is the fewest possible number of times he has been at bat? 
The problem, in a slightly more general setting is (see [2]): given an interval, find in it the rational number with the smallest numerator and denominator.

Gosper's solution is the following: "express the endpoints as continued fractions. Find the first term where they differ and add 1 to the lesser term, unless it is the last. Discard the terms to the right. What is left is the continued fraction for the smallest rational in the interval. (If one fraction terminates but matches the other as far as it goes, append an infinity and proceed as above.)"

This problem gave us the ideas: what if we reverse the question? Given a rational number, $P / Q$, what is the set of real numbers for which $P / Q$ is a best approximation, either of the first kind or the second? Is it an interval or a more complicated set? In the case of best approximations of the first kind, it seems quite natural that this set is an interval. But in the case of the best approximations of the second kind, the remark that follows their definition makes it not so obvious.

We call these sets optimality intervals and the purpose of this paper is to prove that they are intervals indeed. More formally we have the following definition.

DEFINITION 1.3. (a) Given a positive proper rational fraction $P / Q$, we write $\mathscr{O}_{1}(P / Q)$ to denote the set of real numbers to which $P / Q$ is a best approximation of the first kind and (b) $\mathrm{O}_{2}(P / Q)$ to denote the set of real numbers to which $P / Q$ is a best approximation of the second kind.

Before going any further, we need some results from the arithmetic theory of continued fractions.

2. Some results on continued fractions. As usual we write

$$
\left[a_{0} ; a_{1}, a_{2}, \ldots, a_{n}\right]=a_{0}+\frac{1}{a_{1}+\frac{1}{a_{2}+\frac{1}{\ddots+\frac{1}{a_{n}}}}}
$$

and since mostly we are going to stay in $[0,1]$, we drop the integer part $a_{0}$ so that $\left[a_{1}, a_{2}, \ldots, a_{n}\right]$ is to be understood as $\left[0 ; a_{1}, a_{2}, \ldots, a_{n}\right]$.

If continued fractions must represent numbers uniquely, the identity

$$
\left[a_{1}, a_{2}, \ldots, a_{n}, 1\right]=\left[a_{1}, a_{2}, \ldots, a_{n}+1\right]
$$

must be dispensed with, usually by imposing that, apart from number 1 whose continued fraction has only one partial quotient, $a_{0}=1$, all other terminating continued fractions have as a last partial quotient an integer greater than 1 . That is the option adopted in [4].

A different strategy may be chosen which consists in accepting both representations as valid and use one or the other depending on the current setting (see [3, pages 133140]). This is the convention we adopt in this paper.

For nonterminating continued fractions, which represent irrational numbers, no ambiguity arises. 
DEFinition 2.1. We will refer to the set of real numbers in $[0,1]$, whose first $n$ partial quotients are given positive integers $a_{1}, a_{2}, \ldots, a_{n}$ constitute an interval with endpoints: $\left[a_{1}, a_{2}, \ldots, a_{n}\right]$ and $\left[a_{1}, a_{2}, \ldots, a_{n}+1\right]$, as a fundamental interval of rank $n$, or a cylinder. We write $J_{n}\left(a_{1}, a_{2}, \ldots, a_{n}\right)$ to denote such an interval and $\left|J_{n}\right|$ to refer to its length.

With the usual notations for the approximants

$$
\frac{p_{n-1}}{q_{n-1}}=\left[a_{1}, a_{2}, \ldots, a_{n-1}\right], \quad \frac{p_{n}}{q_{n}}=\left[a_{1}, a_{2}, \ldots, a_{n}\right],
$$

it is seen at once that

$$
\left|J_{n}\left(a_{1}, a_{2}, \ldots, a_{n}\right)\right|=\frac{1}{q_{n-1}\left(q_{n-1}+q_{n}\right)} .
$$

Any real number $\omega \in J_{n}\left(a_{1}, a_{2}, \ldots, a_{n}\right)$ admits a representation of the form

$$
\omega=\left[a_{1}, a_{2}, \ldots, a_{n}+\theta\right], \quad \theta \in(0,1] .
$$

The identity

$$
[m+\theta]=\frac{1}{m+\theta}=\frac{1}{m}-\frac{\theta}{m(m+\theta)}
$$

admits the following generalization:

$$
\left[a_{1}, a_{2}, \ldots, a_{n}+\theta\right]=\frac{p_{n}+p_{n-1} \theta}{q_{n}+q_{n-1} \theta}=\frac{p_{n}}{q_{n}}+(-1)^{n-1} \frac{\left(1+\xi_{n}\right) \theta}{1+\xi_{n} \theta}\left|J_{n}\right|
$$

with

$$
\xi_{n}:=\frac{q_{n-1}}{q_{n}}=\left[a_{n}, \ldots, a_{1}\right]
$$

The ratios $\xi_{n}, n=1,2, \ldots$, were introduced by Lévy in his paper on the Gauss-Kuzmin Theorem [6]. For the details see [7, page 10 and pages 155-158].

We will also adhere to the following convention which we state in the form of a definition.

DEFINITION 2.2. Given a positive proper rational fraction $P / Q$, we write

$$
\frac{P}{Q}=\left[a_{1}, a_{2}, \ldots, a_{n}, m\right],
$$

where $n \geq 0$ is an integer and $a_{1}, a_{2}, \ldots, a_{n}, m$ are positive integers. It is to be understood that the case $n=0$ implies that $P / Q$ is the unit fraction $1 / m$ ( $m$ is allowed to take the value 1 ).

Proposition 2.3. Given two positive proper fractions $p / q$ and $P / Q$, the relation

$$
\left|\frac{P}{Q}-\frac{p}{q}\right|=\frac{1}{Q q}
$$


holds if and only if, for some integer $n \geq 0$ there are $n+1$ positive integers, $a_{1}, a_{2}, \ldots$, $a_{n}, m$, which satisfy

$$
\frac{p}{q}=\left[a_{1}, a_{2}, \ldots, a_{n}\right], \quad \frac{P}{Q}=\left[a_{1}, a_{2}, \ldots, a_{n}, m\right]
$$

which amounts to saying that $p / q$ and $P / Q$ are both consecutive approximants to the numbers of the fundamental interval $J_{n+1}\left(a_{1}, a_{2}, \ldots, a_{n}, m\right)$.

REMARK 2.4. If $m=1$ and only in that case $p / q$ and $P / Q$ are the endpoints of an interval of rank $n+1$. In symbols,

$$
\frac{p}{q}=\left[a_{1}, a_{2}, \ldots, a_{n}\right], \quad \frac{P}{Q}=\left[a_{1}, a_{2}, \ldots, a_{n}, 1\right] .
$$

It is perhaps worth to point out that in the simplest case, that of order zero, that is to say when

$$
\frac{p}{q}=\frac{0}{1}=[0 ;]
$$

then, necessarily,

$$
\frac{P}{Q}=\frac{1}{m}=[m]=[m-1,1]
$$

for $m=2,3, \ldots$

When the order is equal to one, which means $p / q=1 / 1=[1]$ then, necessarily,

$$
\frac{P}{Q}=\frac{m-1}{m}=\frac{1}{1+1 /(m-1)}=[1, m-1]
$$

for $m=2,3, \ldots$. The form $\left[a_{1}, a_{2}, \ldots, a_{n}, m\right]$ has been chosen to stress the fact that the same pattern propagates to any order of depth.

For the proof, see [3, Theorem 172, page 140].

LEMMA 2.5. Given the positive proper fraction $P / Q=\left[a_{1}, a_{2}, \ldots, a_{n}, m\right], n \geq 0$, $m \geq 2$, if we set

$$
\frac{p_{n}}{q_{n}}=\left[a_{1}, a_{2}, \ldots, a_{n}\right], \quad \frac{P_{n+1}}{Q_{n+1}}=\left[a_{1}, a_{2}, \ldots, a_{n}, m-1\right],
$$

then, obviously, $q_{n}<Q$ and $Q_{n+1}<Q$ while, except for $P / Q$ itself the rational numbers in the open interval with endpoints $p_{n} / q_{n}$ and $P_{n+1} / Q_{n+1}$ have denominators larger than $Q$.

In other words, $p_{n} / q_{n}$ and $P_{n+1} / Q_{n+1}$ are the closest fractions to $P / Q$ with denominators less than or equal to $Q$. In addition,

$$
\begin{aligned}
\frac{p_{n}}{q_{n}} & <\frac{P}{Q}<\frac{P_{n+1}}{Q_{n+1}}, \quad n=0,2,4, \ldots, \\
\frac{P_{n+1}}{Q_{n+1}} & <\frac{P}{Q}<\frac{p_{n}}{q_{n}}, \quad n=1,3,5, \ldots .
\end{aligned}
$$


Proof. Any number $p / q$ between $p_{n} / q_{n}$ and $P / Q$ may be written as

$$
\left[a_{1}, a_{2}, \ldots, a_{n}, m+k+\theta\right], \quad k \geq 1 \theta \in(0,1),
$$

so that $\left[a_{1}, a_{2}, \ldots, a_{n}, m+k\right]$ is an approximant to $p / q$ and since the sequence of denominators is strictly increasing, then $q>(m+k) q_{n}+q_{n-1} \geq m q_{n}+q_{n-1}=Q$.

On the other hand, the numbers between $P / Q$ and $P_{n+1} / Q_{n+1}$ constitute the fundamental interval $J_{n+1}\left(a_{1}, a_{2}, \ldots, a_{n}, m-1\right)$.

\section{Calculation of $\mathrm{O}_{1}$}

Proposition 3.1. The set of real numbers, to which the positive proper fraction $P / Q=\left[a_{1}, a_{2}, \ldots, a_{n}, m\right]$ is a best approximation of the first kind, is an interval with endpoints

$$
\begin{aligned}
r & :=\left[a_{1}, a_{2}, \ldots, a_{n}, 2 m, a_{n}, \ldots, a_{1}\right], \\
s & :=\left[a_{1}, a_{2}, \ldots, a_{n}, m-1,2, m-1, a_{n}, \ldots, a_{1}\right] .
\end{aligned}
$$

Proof. It is obvious that given two fractions $p / q$ and $P / Q$ which we may suppose ordered from left to right, any real number $\omega$ satisfying

$$
\frac{1}{2}\left(\frac{p}{q}+\frac{P}{Q}\right)<\omega \leq \frac{P}{Q}
$$

is better approximated by $P / Q$.

In view of Lemma 2.5, we only have to show that

$$
\frac{1}{2}\left(\frac{p_{n}}{q_{n}}+\frac{P}{Q}\right)=\left[a_{1}, a_{2}, \ldots, a_{n}, 2 m, a_{n}, \ldots, a_{1}\right] .
$$

Since $r$ belongs to the fundamental interval $J_{n}:=J_{n}\left(a_{1}, a_{2}, \ldots, a_{n}\right)$ it can be written as

$$
r=\left[a_{1}, a_{2}, \ldots, a_{n}+x\right]=\frac{p_{n}}{q_{n}} \pm \frac{\left(1+\xi_{n}\right) x}{1+\xi_{n} x}\left|J_{n}\right|
$$

for some rational $x, 0<x \leq 1$, and $\xi_{n}=\left[a_{n}, \ldots, a_{1}\right]$. Since $P / Q$ may be written as

$$
\frac{P}{Q}=\left[a_{1}, a_{2}, \ldots, a_{n}, m\right]=\frac{p_{n}}{q_{n}} \pm \frac{\left(1+\xi_{n}\right)(1 / m)}{1+\xi_{n}(1 / m)}\left|J_{n}\right|,
$$

the arithmetic mean takes the form

$$
\frac{1}{2}\left(\frac{p_{n}}{q_{n}}+\frac{P}{Q}\right)=\frac{p_{n}}{q_{n}} \pm \frac{1}{2} \frac{\left(1+\xi_{n}\right)}{m+\xi_{n}}\left|J_{n}\right|
$$

and equating the two expressions,

$$
\frac{x}{1+\xi_{n} x}=\frac{1}{2\left(m+\xi_{n}\right)} .
$$


A simple calculation shows that the last equation is equivalent to

$$
x=\frac{1}{2 m+\xi_{n}}
$$

and since $\xi_{n}=\left[a_{n}, \ldots, a_{1}\right]$ we finally get

$$
x=\left[2 m, a_{n}, \ldots, a_{1}\right] \Longleftrightarrow r=\left[a_{1}, a_{2}, \ldots, a_{n}, 2 m, a_{n}, \ldots, a_{1}\right]
$$

As to the other endpoint $s$, again in view of Lemma 2.5 setting

$$
J_{n+1}:=J_{n+1}\left(a_{1}, a_{2}, \ldots, a_{n}, m-1\right),
$$

the same argument applies and now we have

$$
s:=\frac{1}{2}\left(\frac{P_{n+1}}{Q_{n+1}}+\frac{P}{Q}\right)=\frac{P_{n+1}}{Q_{n+1}} \pm \frac{1}{2}\left|J_{n+1}\right|
$$

which, as the midpoint of $J_{n+1}$, can also be written as

$$
\left[a_{1}, a_{2}, \ldots, a_{n}, m-1, y\right]=\frac{P_{n+1}}{Q_{n+1}} \pm \frac{\left(1+\xi_{n+1}\right) y}{1+\xi_{n+1} y}\left|J_{n+1}\right|
$$

for some rational $y, 0<y \leq 1$, and $\xi_{n+1}=\left[m-1, a_{n}, \ldots, a_{1}\right]$. Equating (3.11) and (3.12), we get

$$
\frac{1}{2}=\frac{\left(1+\xi_{n+1}\right) y}{1+\xi_{n+1} y}
$$

which is easily seen to imply

$$
y=\frac{1}{2+\xi_{n+1}}=\left[2, m-1, a_{n}, \ldots, a_{1}\right]
$$

and finally

$$
s=\left[a_{1}, a_{2}, \ldots, a_{n}, m-1,2, m-1, a_{n}, \ldots, a_{1}\right]
$$

Thus Proposition 3.1 is proved.

4. Calculation of $\mathrm{O}_{2}$. For $P / Q$ falling short of being an optimal approximation to the real number $\omega$ there must exist a fraction $p / q$ with $q \leq Q$ and

$$
|q \omega-p| \leq|Q \omega-P|,
$$

which might be phrased saying that $p / q$ prevents $P / Q$ from being an optimal approximation to $\omega$. 
The following remarks show that when studying approximations of the second kind, mediant fractions play the role formerly played by arithmetic means. If we suppose for instance, that $p / q<\omega<P / Q$, then condition (4.1) amounts to

$$
(q+Q) \omega \leq p+P \Longleftrightarrow \omega \leq \frac{p+P}{q+Q},
$$

while in the case that $p / q<P / Q<\omega$, condition (4.1) is equivalent to

$$
(P-p) \omega \geq Q-q \Longleftrightarrow \omega \geq \frac{P-p}{Q-q} .
$$

To sum up, if $p / q<P / Q$, the condition for $p / q$ not to prevent $P / Q$ from being an optimal approximation to $\omega$ is that $\omega$ belongs to the open interval

$$
I_{l}:=\left(\frac{p+P}{q+Q}, \frac{P-p}{Q-q}\right),
$$

while if $P / Q<p / q$, the condition is

$$
\omega \in\left(\frac{P-p}{Q-q}, \frac{p+P}{q+Q}\right)=: I_{r} .
$$

Since no ambiguity may arise, we agree to write $\Phi(p / q \rightarrow P / Q)$ to refer to $\Phi_{l}$ or $\Phi_{r}$ depending on which side $p / q$ lies. With this notation it becomes obvious that

$$
\mathrm{O}_{2}\left(\frac{P}{Q}\right)=\bigcap_{p \leq q \leq Q} g\left(\frac{p}{q} \longrightarrow \frac{P}{Q}\right)
$$

as $\omega \in \mathrm{O}_{2}(P / Q)$ if and only if $\omega$ belongs to all possible $\mathscr{I}(p / q \rightarrow P / Q)$. This, in addition, proves that $\mathrm{O}_{2}(P / Q)$ is an interval. The following lemma, where we switch to determinant notation, does most of the job.

LEMMA 4.1. Let $a / b, p / q, P / Q$ be three positive proper fractions.

(a) If $a / b<p / q<P / Q$, then the condition

$$
\frac{P}{Q}-\frac{p}{q}=\frac{1}{Q q} \Longleftrightarrow\left|\begin{array}{ll}
P & p \\
Q & q
\end{array}\right|=1
$$

implies

$$
\frac{a+P}{b+Q} \leq \frac{p+P}{q+Q}, \quad \frac{P-p}{Q-q} \leq \frac{P-a}{Q-b} .
$$

(b) In a similar way, if $P / Q<p / q<a / b$, then the condition

$$
\frac{p}{q}-\frac{P}{Q}=\frac{1}{Q q} \Longleftrightarrow\left|\begin{array}{ll}
p & P \\
q & Q
\end{array}\right|=1
$$

implies

$$
\frac{p+P}{q+Q} \geq \frac{a+P}{b+Q}, \quad \frac{P-a}{Q-b} \leq \frac{P-p}{Q-q} .
$$


In other words, in any case

$$
\Im\left(\frac{p}{q} \longrightarrow \frac{P}{Q}\right) \subseteq g\left(\frac{a}{b} \longrightarrow \frac{P}{Q}\right) .
$$

Proof. We only give the details of case (a). It is sufficient to show that

$$
\begin{aligned}
& \left|\begin{array}{ll}
p+P & a+P \\
q+Q & b+Q
\end{array}\right| \geq 0, \\
& \left|\begin{array}{ll}
P-a & P-p \\
Q-b & Q-q
\end{array}\right| \geq 0 .
\end{aligned}
$$

Since (4.7) implies

$$
\left|\begin{array}{ll}
p+P & P \\
q+Q & Q
\end{array}\right|=\left|\begin{array}{ll}
P-p & P \\
Q-q & Q
\end{array}\right|=-1
$$

from

$$
\left|\begin{array}{ll}
p+P & a+P \\
q+Q & b+Q
\end{array}\right|=\left|\begin{array}{ll}
p+P & a \\
q+Q & b
\end{array}\right|+\left|\begin{array}{cc}
p+P & P \\
q+Q & Q
\end{array}\right|=\left|\begin{array}{cc}
p+P & a \\
q+Q & b
\end{array}\right|-1
$$

and the fact that $\left|\begin{array}{ll}p+P & a \\ q+Q & b\end{array}\right|$ is a positive integer, we get

$$
\left|\begin{array}{ll}
p+P & a+P \\
q+Q & b+Q
\end{array}\right| \geq 1-1=0
$$

which proves case (4.12).

In an analogous way, from

$$
\left|\begin{array}{ll}
P-a & P-p \\
Q-b & Q-q
\end{array}\right|=\left|\begin{array}{ll}
P & P-p \\
Q & Q-q
\end{array}\right|+\left|\begin{array}{cc}
-a & P-p \\
-b & Q-q
\end{array}\right|=-1+\left|\begin{array}{ll}
P-p & a \\
Q-q & b
\end{array}\right|
$$

Since, obviously $a / b<P / Q<(P-p) /(Q-q)$, again $\left|\begin{array}{cc}P-p & a \\ Q-q & b\end{array}\right|$ is a positive integer and (4.13) follows.

Proposition 4.2. The set of real numbers, to which the positive proper fraction $P / Q=\left[a_{1}, a_{2}, \ldots, a_{n}, m\right]$ is a best approximation of the second kind, is an interval with endpoints

$$
\begin{aligned}
r & :=\left[a_{1}, a_{2}, \ldots, a_{n}, m+1\right]=\left[a_{1}, a_{2}, \ldots, a_{n}, m, 1\right], \\
s & :=\left[a_{1}, a_{2}, \ldots, a_{n}, m-1,2\right] .
\end{aligned}
$$

Proof. By Lemma 2.5, $p_{n} / q_{n}$ and $P_{n+1} / Q_{n+1}$ are the closest fractions to $P / Q$ with denominators less than or equal to $Q$ and, obviously verify (4.7). Lemma 4.1 implies that no other fraction may prevent $P / Q$ from being an optimal approximation to the numbers of the set

$$
\mathscr{I}\left(\frac{p_{n}}{q_{n}} \longrightarrow \frac{P}{Q}\right) \cap \Im\left(\frac{P_{n+1}}{Q_{n+1}} \longrightarrow \frac{P}{Q}\right)
$$


which is easily seen to be the interval with endpoints of the mediant fractions

$$
\frac{p_{n}+P}{q_{n}+Q}, \quad \frac{P_{n+1}+P}{Q_{n+1}+Q} .
$$

Two further calculations show that

$$
\begin{gathered}
\frac{p_{n}+P}{q_{n}+Q}=\frac{(m+1) p_{n}+p_{n-1}}{(m+1) q_{n}+q_{n-1}}, \\
\frac{P_{n+1}+P}{Q_{n+1}+Q}=\frac{(2 m-1) p_{n}+2 p_{n-1}}{(2 m-1) q_{n}+2 q_{n-1}}=\frac{(m-1+1 / 2) p_{n}+p_{n-1}}{(m-1+1 / 2) q_{n}+q_{n-1}},
\end{gathered}
$$

which prove (4.18).

\section{REFERENCES}

[1] C. Brezinski, History of Continued Fractions and Padé Approximants, Computational Mathematics, vol. 12, Springer-Verlag, Berlin, 1991.

[2] R. W. Gosper, Continued fractions, preprint, 1972, http://www.inwap.com/pdp10/hbaker/ hakmem/cf.html\#item101c.

[3] G. H. Hardy and E. M. Wright, An Introduction to the Theory of Numbers, 5th ed., The Clarendon Press, Oxford University Press, New York, 1979.

[4] A. Y. Khintchine, Continued Fractions, P. Noordhoff Ltd., Groningen, 1963.

[5] D. E. Knuth, Seminumerical Algorithms, 2nd ed., The Art of Computer Programming, vol. 2, Addison-Wesley, Massachusetts, 1981.

[6] P. Lévy, Sur les lois de probabilité dont dépendent les quotients complets et incomplets d'une fraction continue, Bull. Soc. Math. France 57 (1929), 178-194 (French).

[7] A. M. Rockett and P. Szüsz, Continued Fractions, World Scientific, New Jersey, 1992.

Lluís Bibiloni: FACultat de CiÈnCies de l'Educació, Universidad Autònoma DE BARCELONA, 08193 BellaterRa, BARCELONA, SPAin

E-mail address: 1.bibi1oni@uab.es

Pelegrí Viader: Departament D’EConomia i Empresa, Universidad Pompeu Fabra, RAMON TRIAS FARGAS 25-27, 08005 BARCELONA, SPAIN

E-mail address: pelegri .viader@econ.upf.es

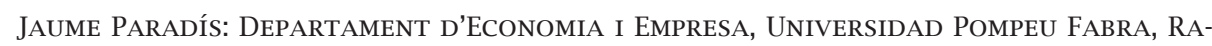
MON TRIAS FARGAS 25-27, 08005 BARCELONA, SPAIN

E-mail address: jaume.paradis@econ.upf.es 


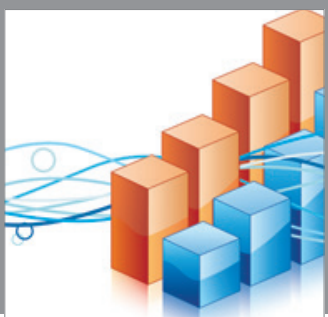

Advances in

Operations Research

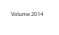

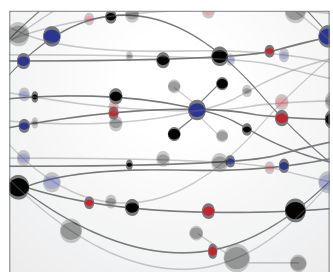

\section{The Scientific} World Journal
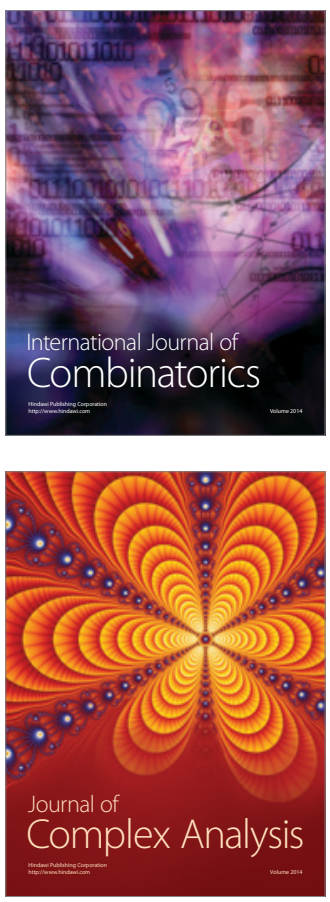

International Journal of

Mathematics and

Mathematical

Sciences
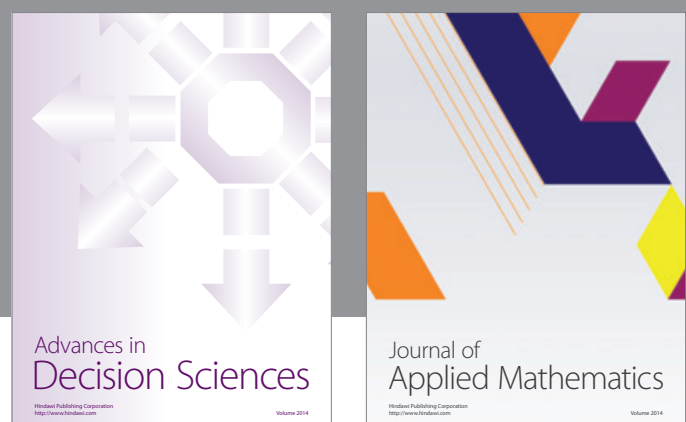

Journal of

Applied Mathematics
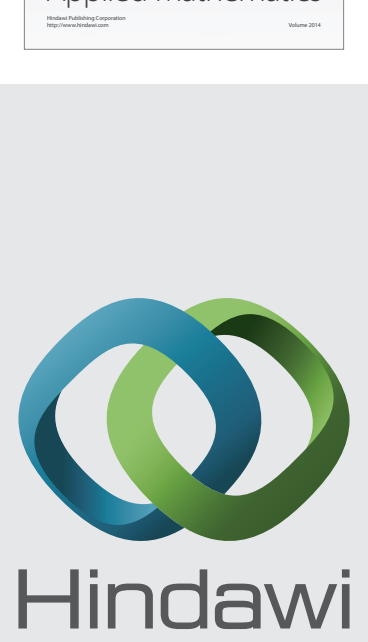

Submit your manuscripts at http://www.hindawi.com
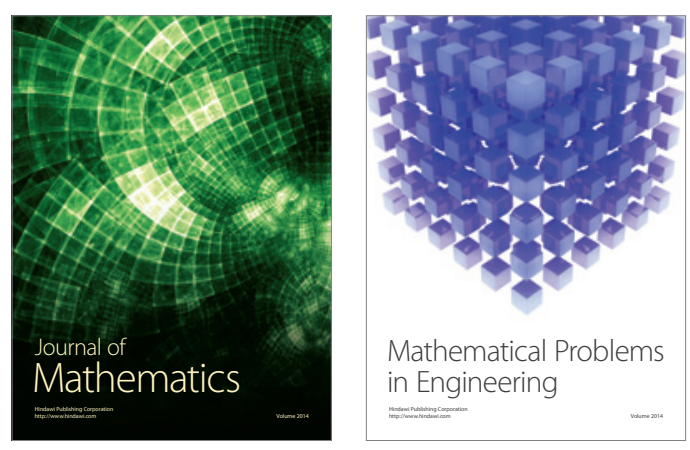

Mathematical Problems in Engineering
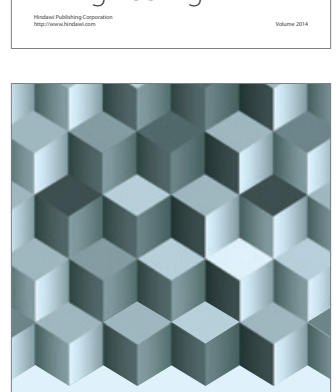

Journal of

Function Spaces
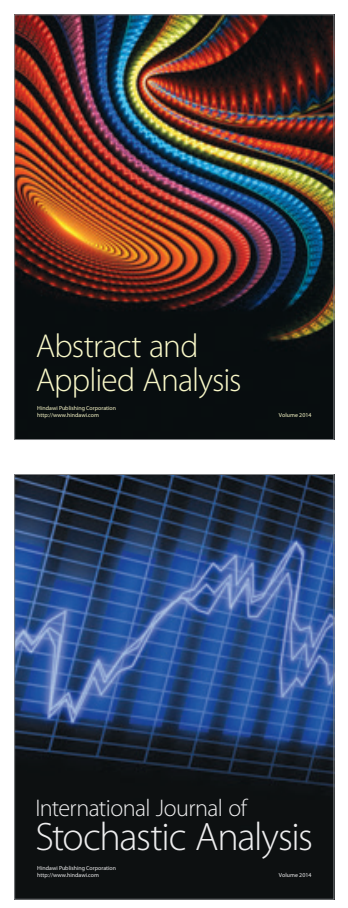

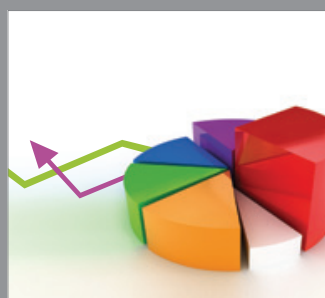

ournal of

Probability and Statistics

Promensencen
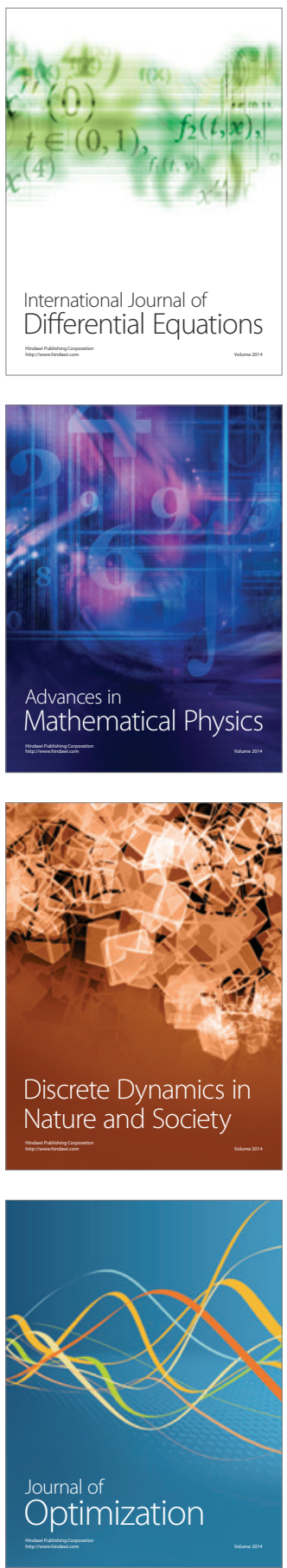